Research Paper

\title{
hsa_circ_0071106 can be used as a diagnostic marker for type 2 diabetes
}

\author{
Zheng Yingying ${ }^{*}$, Yu Yongij $3^{*}$, Cheng Qiuting ${ }^{1}$, Liao Rifang ${ }^{\circledR}$ and Zeng Zhuanping ${ }^{\bowtie}$ \\ 1. School of Public Health, Guangdong Pharmaceutical University, Guangzhou 510310, China. \\ 2. Department of Pharmacy, Sun Yat-Sen Memorial Hospital, Sun Yat-sen University, Guangzhou 510120, China. \\ 3. The Second People's Hospital of Huadu District, Guangzhou 510320, China. \\ ${ }^{*}$ Co-first authors. \\ $\triangle$ Corresponding authors: Zeng Zhuanping, E-mail: 1141518464@qq.com; and Liao Rifang, E-mail: liaorf@mail.sysu.edu.cn.
}

(1) The author(s). This is an open access article distributed under the terms of the Creative Commons Attribution License (https://creativecommons.org/licenses/by/4.0/). See http://ivyspring.com/terms for full terms and conditions.

Received: 2020.09.03; Accepted: 2020.12.18; Published: 2021.04 .03

\begin{abstract}
We explored hsa_circ_0071106 as a diagnostic marker for type 2 diabetes (T2DM) in south China, and predicted the functional mechanism of the target circRNA. A total of 107 T2DM patients and 107 healthy reference persons were included as the research objects. In the first stage, the circRNA microarray was used to detect the peripheral blood of 4 T2DM and 4 control groups to screen the differential expression profile of circRNA. In the second stage, four circRNAs were screened from the differential expression profiles of circRNA, and real-time polymerase chain reaction (Q-PCR) technology was used to verify the blood samples of 103 T2DM and 103 controls. Finally, Gene Ontology (GO) and Kyoto Encyclopedia of Genes and Genomes (KEGG) pathway analysis in bioinformatics were used to predict the functional mechanism of target circRNA. Lastly, we found that hsa circ 0071106 increase the risk of T2DM $(\mathrm{OR}=2.819$ (95\% Cl: 1.415 5.616)). The area under the ROCCurve hsa_circ_0071106 was 0.690, the sensitivity was $62.1 \%$, and the specificity was $69.9 \%$. The function prediction results showed that hsa_circ_0071106 was involved in biological processes such as protein binding, gene transcription, and may be involved in the pathway of hsa-miR-29a-5p regulating diabetes, hsa_circ_0071106 may be used as a diagnostic marker for T2DM.
\end{abstract}

Key words: diagnostic marker, type 2 diabetes, circRNA microarray

\section{Introduction}

According to the International Diabetes Federation (IDF) Diabetes Atlas (9th Edition, 2019), nearly 463 million diabetic patients exist worldwide, 1 in 2 adults with diabetes are undiagnosed [1]. By 2040, the number of patients with diabetes may increase to 642 million [2,3]. In the advanced stages of T2DM, patients often experience various complications, and it will also bring a very heavy financial burden [4]. Therefore, early diagnosis and intervention are urgently needed. There are currently three methods for diagnosing diabetes. The oral glucose tolerance test (OGTT) is the gold standard for clinical detection of T2DM [5], but the process is time-consuming and complicated, fasting blood glucose (FPG) detection method is convenient, but its error rate is high [6], the detection of glycated hemoglobin (HbA1c) has not been standardized in China. Therefore, current diagnosis measures for T2DM show various insufficiencies, the above methods are considered for diagnosis and treatment when the suspect is suspected of having T2DM. When the patient is confirmed as T2DM, The critical period of abnormal glucose tolerance that can reverse diabetes has been missed [7]. So it is necessary to explore early biomarkers with high specificity, high sensitivity and fast test speed for T2DM, which is conducive to the early prevention and treatment of T2DM.

With the maturation of molecular biology technologies such as high-throughput sequencing and the implementation of the Human Genome Project. In recent years, circRNA has been found to be a diagnostic marker for various diseases such as the 
cardiovascular system [8-9], nervous system [10-11] and tumors [12-13]. CircRNA has the characteristics of structural stability [14], tissue specificity [15], evolutionary conservatism [16], and it is difficult for exonuclease to degrade it [17], and has sponge-like adsorption to inhibit the activity of microRNA [14], regulate gene transcription [18], bind RNA binding protein, participate in translation protein and other functions [19], these characteristics and functions have contributed to circRNA as a new biomarker and the target of disease treatment for the diagnosis of noninfectious chronic diseases such as diabetes. Therefore, in this study, the circRNA microarray analysis is used to screen out the significantly different circRNA expression profiles in T2DM, then the Q-PCR technology is used to verify reliability in the expanded population, and finally the target circRNA is analyzed through bioinformatics to predict the target genes and possible signal pathways related to T2DM, and provide a basis for the prevention and control measures of T2DM.

\section{Research design and methods}

\section{Research object}

The research object of this study comes from the healthy screening population over 18 years of age in Huadu District, Guangzhou, from January to October 2019. According to the $1: 1$ individual matching case control study design, the matching conditions are the same gender and community and age less than 5 years old, a total of 107 diabetic patients and 107 healthy controls were included. In the first stage, 4 pairs of samples were selected for circRNA microarray test to screen for differential expression profiles. In the second stage, 103 pairs of samples were taken for Q-PCR verification. And the Ethics Committee of Guangdong Pharmaceutical University approved the study.

\section{Inclusion criteria}

\section{Diabetes group}

According to the "China T2DM Prevention and Treatment Guidelines (2017 Version)" [20] standard grouping: fasting blood glucose value $\geq 7.0 \mathrm{mmol} / \mathrm{L}$ or $2 \mathrm{~h}$ postprandial blood glucose value $\geq 11.1 \mathrm{mmol} / \mathrm{L}$ or glycated hemoglobin $\mathrm{HbA} 1 \mathrm{c} \geq 6.5 \%$ or a previous history of diabetes or have typical symptoms of diabetes such as multiple drinks, polyphagia, polyuria and unexplained weight loss, with random blood glucose values $\geq 11.1 \mathrm{mmol} / \mathrm{L}$.

\section{Control group}

Healthy people with fasting blood glucose value $<7.0 \mathrm{mmol} / \mathrm{L}$ and random blood glucose value $<11.1 \mathrm{mmol} / \mathrm{L}$ and 2 hours postprandial blood glucose value $<11.1 \mathrm{mmol} / \mathrm{L}$ in the same sex and same area and 5 years old as diabetic patients were selected as the control group.

\section{Exclusion criteria}

Diabetes group and control group excluded patients with other types of diabetes such as type 1 diabetes, gestational diabetes, and patients with cerebral infarction, myocardial infarction, malignant tumors, and chronic inflammation.

\section{Whole blood sample collection and total RNA extraction}

The subjects collected $3 \mathrm{~mL}$ of whole blood using a vacuum blood collection tube containing EDTA anticoagulant in the morning on an empty stomach. Immediately freeze the samples and transport them to the ultra-low temperature refrigerator through dry ice to store them for subsequent sample extraction. RNAiso Blood (Takara Bio Inc, Japan) extraction reagent was used to extract total RNA from $300 \mathrm{ul}$ of whole blood, Nano Drop-2000 was used to determine the concentration and purity of total RNA, and the integrity of total RNA was using modified agarose gel electrophoresis for detection.

\section{CircRNA microarray analysis}

During the experiment, Arraystar Human circRNA Arrays v2 provided by Arraystar was used to extract the peripheral blood of 4 diabetes and 4 control groups for microarray analysis. The circRNA microarray used ribonucleic acid $R$ (Rnase $R$ ) to decompose the total RNA into linear RNA and circular RNA, removed the linear RNA in the sample, enriched the circular RNA in the sample. Then the enriched circRNAs were amplified according to the random primer method and transcribed into cRNA with a fluorescent substance label, and then sequentially hybridized to the circRNA microarray according to the principle of nucleic acid hybridization. After the hybridization, the position of the reaction point and the fluorescence intensity are scanned using a chip scanner, and then imported into the relevant analysis software for data analysis. Differentially expressed circRNAs between two samples were identified through Fold Change, $|\mathrm{FC}| \geq 1.5$ and $t$ test, $P<0.05$ filtering.

\section{Q-PCR validates differentially expressed circRNAs}

Four circRNAs were selected from circRNA differential expression profiles for population Q-PCR experiments. Both the Prime Script ${ }^{\mathrm{TM}} \mathrm{RT}$ reagent Kit with gDNA Eraser and Q-PCR kits were equipped with Takara Bio Inc, Japan. Reverse transcription 
reaction system: $5 \times$ gDNA Eraser Buffer 2.0ul, gDNA Eraser $1.0 \mathrm{ul}$, add appropriate amount of total RNA and make up to $10 \mathrm{ul}$ with enzyme-free water, place at $42{ }^{\circ} \mathrm{C}$ and heat for 2 minutes to remove the DNA from the genome, then add to the above liquid PrimeScript RT Enzyme Mix I 1.0 ul, RT Primer Mix 1.0 ul, 5× PrimeScript Buffer 2 (for Real Time) 4.0 ul, RNase Free $\mathrm{dH}_{2} \mathrm{O} 4.0 \mathrm{ul}$, shake gently and immediately put at 37 ${ }^{\circ} \mathrm{C}$ for 15 minutes. The extension reaction was carried out under the conditions of reverse transcription inactivation in an environment of $85^{\circ} \mathrm{C}$ and 5 seconds, and finally it was cooled under the heat preservation condition of 4. Q-PCR reaction system: TB Green Premix Ex Taq (Tli RNaseH Plius) (2X) 10 ul, PCR Forward Primer (10M) 0.8 ul, PCR Reverse Primer (10 M) $0.8 \mathrm{ul}$, ROX Reference Dye (20X) 0.4 ul, enzymefree water 6 ul, cDNA 2 ul. Reaction conditions: $95^{\circ} \mathrm{C}$, $34 \mathrm{~s}$ cycle to activate enzyme activity once, $95^{\circ} \mathrm{C}, 5 \mathrm{~s}$, $60{ }^{\circ} \mathrm{C}$, $34 \mathrm{~s}$ for 40 cycles of extension reaction, using housekeeping gene GAPDH as internal reference, and finally set the melting curve analysis. Table 1 is the primer sequence of target circRNA and GAPDH.

Table 1. Primer sequences of target circRNA and GAPDH

\begin{tabular}{ll}
\hline Primers & Amplified sequence \\
\hline hsa_circ_0071271-F & 5'-GGAACCCAAAGACCTGCTACAA-3' \\
hsa_circ_0071271-R & 5'-TGGTCCACTCCAGCTCCTCT-3' \\
hsa_circ_0003344-F & 5'-GGAAGAGCTACGGGAGATCAA-3' \\
hsa_circ_0003344-R & 5'-CCAGGTCTCCCTTATCGACCT-3' \\
hsa_circ_0000284-F & 5'-ATAGACTTTGGGTCGGCCAGT-3' \\
hsa_circ_0000284-R & 5'-TCTTCACACTACAAAAGGCACTTGA-3' \\
hsa_circ_0071106-F & 5'-GAAGCTGCTGATCGGAAGAAA-3' \\
hsa_circ_0071106-R & 5'-GCCGGTTCTGCTCTACTTGG-3' \\
GAPDH-F & 5'-GCACCGTCAAGGCTGAGAAC-3' \\
GAPDH-R & 5'-TGGTGAAGACGCCAGTGGA-3' \\
\hline
\end{tabular}

\section{Statistical analysis}

The collected basic data and Q-PCR experimental data were integrated into the software SAS 9.4 for analysis. When the quantitative data presents the normal distribution of the data, the means \pm standard deviation is used to represent, and the continuity data that does not present the normal distribution is represented by the median number and the quarter-value interval. The difference analysis of quantitative data between two groups was by paired $t$ test or Wilcoxon signed-rank test, and the distribution difference of demographic characteristics and life behavior factors between diabetes group and control group was by paired $x^{2}$ test. The diagnostic value of circRNA in diabetes needs to be evaluated using the ROC curve analysis, calculating the area under the curve (AUC), sensitivity, specificity, maximum Youden index and the best cut-off point. The AUC $<0.5$ has no diagnostic value and AUC $>0.5$ has diagnostic value. To further analyze the relationship between target circRNA and diabetes, a multifactor conditional logistic regression model with correction for confounding factors was used for analysis.

\section{Results}

\section{CircRNA microarray analysis results}

The total RNA concentration in the peripheral blood of 4 pairs of diabetic group and the control group was 160 550 ng/ul, and the purity was between 1.8 2.3. The total RNA quality met the requirements of the circRNA microarray analysis. The modified agarose gel electrophoresis was used to detect the integrity of the total RNA. The $28 \mathrm{~s}$ and $18 \mathrm{~s}$ bands of the total RNA were clear, and the $5 \mathrm{~s}$ band was vague, indicating that there was no obvious non-specific amplification band and primer dimer in the product band (Figure 1).

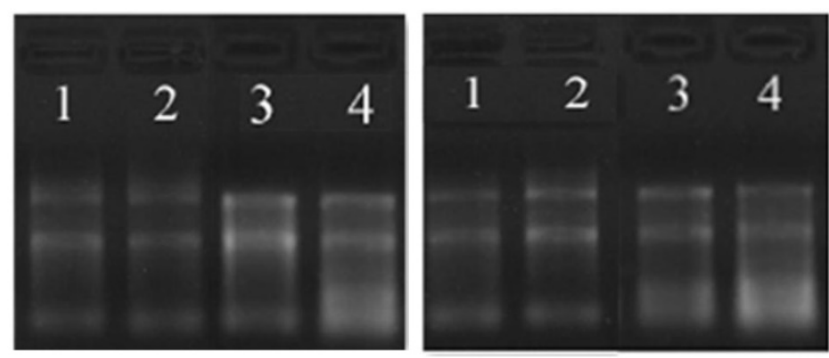

Figure 1. RNA agarose gel electrophoresis (the left picture is the diabetes group, the right picture is the control group).

\section{Volcano and scatter plots}

The log value of the $\log _{2}$ logarithmic conversion of the average expression of circRNA in the control group was plotted on the abscissa, and the log value of the $\log 2$ logarithmic conversion of the average expression of circRNA in the diabetes group was plotted on the ordinate (Figure 2). In the figure, the part above the line1 and below the line 2 was $|F C| \geq 1.5, \quad P<0.05$, the significant difference in expression of circRNA. Above line 1 was up-regulated circRNA, and below line 2 was down-regulated circRNA.

The volcano graph was plotted according to the FC and $P$ value. The abscissa was $\log 2$ (FC) and the ordinate was $-\log 10$ ( $P$ value) to create a volcano graph (Figure 3 ). In the figure, the part above the horizontal green line and beyond the two vertical green lines indicated the distribution of circRNA with $|F C| \geq 1.5$ and $P<0.05$. The red dots in the figure indicated the differential circRNA, some in the upper left corner indicated the down-regulated circRNA, and those in the upper right corner indicated the up-regulation circRNA. 


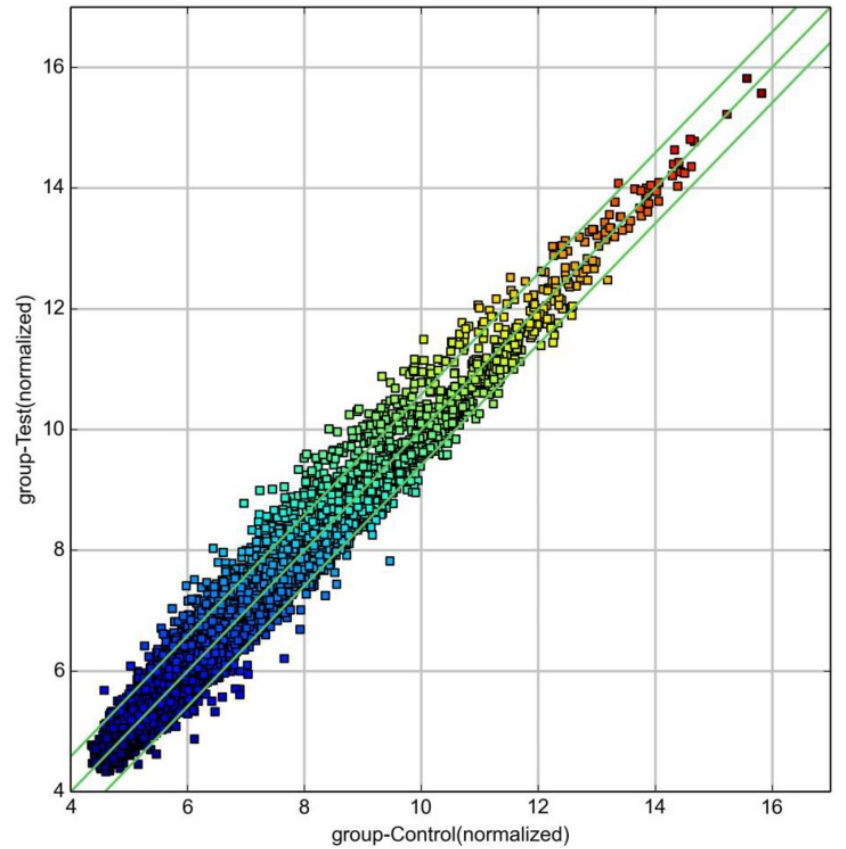

Figure 2. circRNA scatter plot (diabetes group and control group).

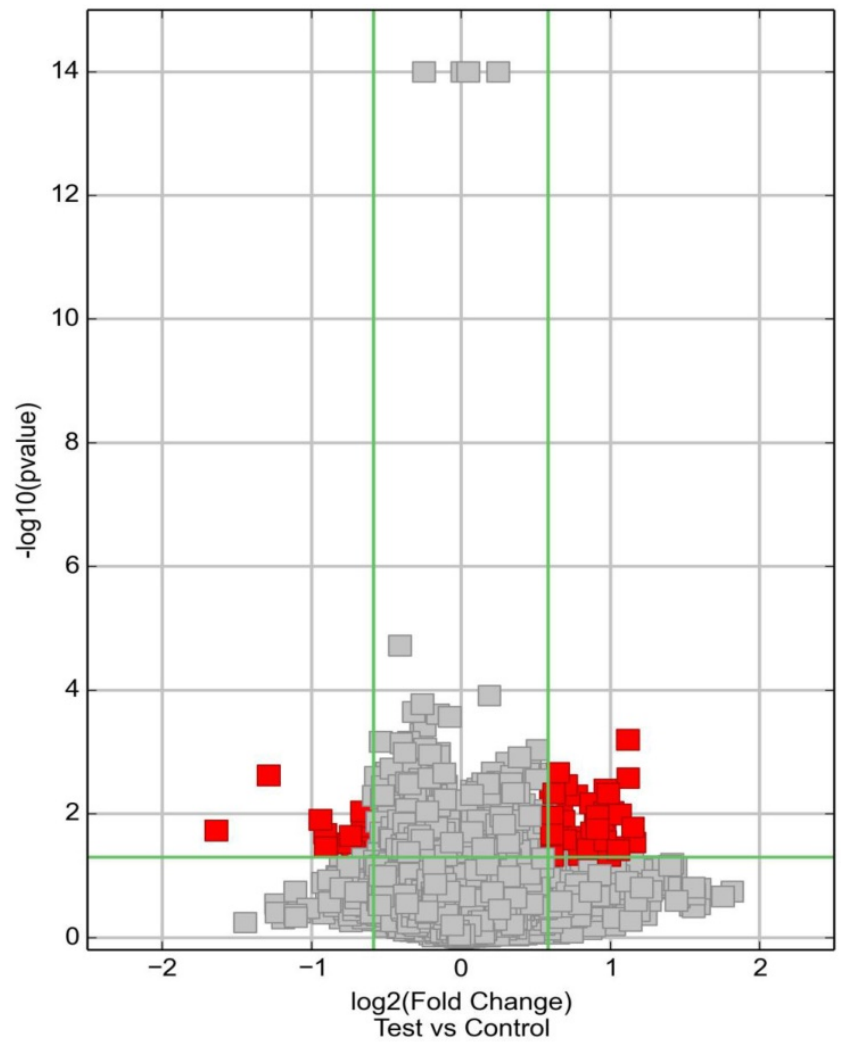

Figure 3. circRNA volcano graph (diabetes group and control group).

\section{CircRNA differential expression profile}

A total of 798 circRNAs were screened from the circRNA microarray results, of which 296 were upregulated and 502 were down-regulated. However, according to the $|F C| \geq 1.5$ and $P<0.05$, the results showed that a total of 86 circRNAs was differential expression, of which 70 expressions were upregulated and 16 were down-regulated. The candidate biomarkers were selected from the 86 circRNAs utilizing stricter screening criteria: $\mathrm{P}<0.01,|\mathrm{FC}| \geq 2$, exon and the original intensity is greater than 200 . Four candidate circRNAs met these criteria: hsa_circ_0071106, hsa_circ_0071271, hsa_circ_0000284 and hsa_circ_0003344 (Table 1).

\section{The basic characteristics of the research object of Q-PCR experiment}

To validate the candidate circRNAs, Q-PCR was conducted in the 103 pairs of subjects. There were 71 males and 32 females in the diabetes group with an age distribution of $50.71 \pm 12.57$ years old and 71 males and 32 females in the control group with an age distribution of $50.22 \pm 12.68$ years old. The distribution of age groups showed that the middle-aged group (45-59 years old) accounts for the largest proportion, followed by the youth group (18-44 years old), and the elderly group was less distributed in this study. Table 2 describes the demographic characteristics of the study subjects. Among them, occupational status and hyperlipidemia were statistically significant between the diabetes group and the control group, while the age group, smoking, drinking, and hypertension were not statistically different between the two groups.

Table 2. Demographic characteristics of the research object

\begin{tabular}{|c|c|c|c|c|}
\hline Variable & Control $(n=103)$ & T2DM (n=103) & $\chi^{2}$ & $P$ \\
\hline Age & & & 0.047 & 0.977 \\
\hline $18 \sim$ & $31(30.10)$ & $30(29.13)$ & & \\
\hline $45 \sim$ & $56(54.37)$ & $56(54.37)$ & & \\
\hline $60 \sim$ & $16(15.53)$ & $17(16.50)$ & & \\
\hline Occupation & & & 14.842 & 0.002 \\
\hline Unemployment & $34(33.01)$ & $60(58.25)$ & & \\
\hline Business people & $7(6.8)$ & $3(2.91)$ & & \\
\hline Farmer & $11(10.68)$ & $11(10.68)$ & & \\
\hline Industrialist & $51(49.51)$ & $29(28.16)$ & & \\
\hline Smoke & & & 0.951 & 0.330 \\
\hline No & 75 (72.82) & 81 (78.64) & & \\
\hline Yes & $28(27.18)$ & $22(21.36)$ & & \\
\hline Drink & & & 0.126 & 0.723 \\
\hline No & $86(83.5)$ & 87 (85.29) & & \\
\hline Yes & $17(16.5)$ & 15 (14.71) & & \\
\hline Hyperlipidemia & & & 9.690 & 0.002 \\
\hline $\mathrm{No}$ & 91 (55.49) & $12(28.57)$ & & \\
\hline Yes & $73(44.51)$ & $30(71.43)$ & & \\
\hline Hypertension & & & 1.215 & 0.270 \\
\hline No & $79(52.32)$ & $24(43.64)$ & & \\
\hline Yes & $72(47.68)$ & $31(56.36)$ & & \\
\hline
\end{tabular}

Table 3 compares the expression levels of biochemical indexes between the diabetic group and the control group. Because the data distribution type was skewed, the Wilcoxon signed-rank test was used. Results showed that aspartate aminotransferase (AST), blood glucose (Glu), glycated hemoglobin 
(HbA1c), low-density cholesterol (LDL), triglyceride (TG), total cholesterol (TC), sodium $(\mathrm{Na})$, chlorine $(\mathrm{Cl})$, those indexes were statistically different between diabetes and the control group, while diastolic blood pressure (DBP), systolic blood pressure (SBP), alanine aminotransferase (ALT), high-density cholesterol (HDL), potassium (K), calcium $(\mathrm{Ga})$, which was no statistically significant difference in the indexes between diabetes and the control group.

Table 3. Comparison of the expression levels of biochemical indexes between the diabetes group and the control group

\begin{tabular}{lllll}
\hline Variable & Control & T2DM & $Z$ & $P$ \\
\hline SBP $(\mathrm{mmHg})$ & $134(123,151)$ & $138(125,157)$ & 274.0 & 0.370 \\
DBP (mmHg) & $82(74,94)$ & $85(75,94)$ & 201.0 & 0.486 \\
ALT (IU/L) & $20(15,38)$ & $24(17,39)$ & 23.5 & 0.936 \\
AST (IU/L) & $20.6(17,31)$ & $19(15.2,23)$ & -931.5 & 0.002 \\
Glu & $5.6(5.2,6.2)$ & $15.8(10.8,22.5)$ & 2516.5 & $<0.001$ \\
HbA1c & $5.4(5.1,5.7)$ & $10.4(9.0,11.9)$ & 2678.0 & $<0.001$ \\
LDL (mmol/L) & $2.89(2.53,3.29)$ & $3.22(2.74,3.86)$ & 862.5 & 0.004 \\
HDL (mmol/L) & $1.36(1.19,1.50)$ & $1.37(1.21,1.56)$ & 388.0 & 0.197 \\
TG (mmol/L) & $1.45(0.98,3.64)$ & $2.82(2.10,4.65)$ & 1795.0 & $<0.001$ \\
TC $(\mathrm{mmol} / \mathrm{L})$ & $4.60(4.04,5.20)$ & $5.39(4.80,6.10)$ & 1278.0 & $<0.001$ \\
K & $3.89(3.68,4.14)$ & $3.97(3.69,4.16)$ & 233.5 & 0.438 \\
Ca & $2.33(2.24,2.41)$ & $2.35(2.24,2.40)$ & -10.0 & 0.972 \\
Na & $138.90(135.20,141.00)$ & $134.10(128.20,137.60)$ & -1050.5 & $<0.001$ \\
Cl & $103.50(101.5,106.0)$ & $99.5(98.0,101.9)$ & -2060.0 & $<0.001$ \\
\hline
\end{tabular}

\section{Analysis of relative expression of target circRNA}

The data processing of Q-PCR was analyzed by relative quantitative calculation method. The results showed that hsa_circ_0071106 had a statistically significant difference between the diabetic group and the control group, while hsa_circ_0000284, hsa_circ_ 0003344, and hsa_circ_0071271 had no statistically significant difference between the two groups. In addition, the $\Delta \mathrm{Ct}$ value of hsa_circ_0071106, hsa_circ_ 0071271, hsa_circ_0000284 from the control group to the diabetes group is decreasing, indicating that the expression level of hsa_circ_0071106, hsa_circ_ 0071271, hsa_circ_0000284 in the diabetes group was higher than that in the control group. The $\Delta \mathrm{Ct}$ value of hsa_circ_0003344 from the control group to the diabetes group was rising, that indicated the expression level of the diabetic group was lower than that of the control group, which was consistent with the results of the microarray analysis (Table 4).

Table 4. Verification of relative expression of target circRNA in diabetes and control group by qPCR

\begin{tabular}{lllll}
\hline Target circRNA & Control & T2DM & $t$ & $P$ \\
\hline hsa_circ_0071106 & $5.58 \pm 0.75$ & $5.34 \pm 0.71$ & 2.65 & 0.009 \\
hsa_circ_0000284 & $2.35 \pm 0.75$ & $2.29 \pm 0.65$ & 0.66 & 0.508 \\
hsa_circ_0003344 & $4.25 \pm 1.29$ & $4.30 \pm 1.17$ & 0.32 & 0.751 \\
hsa_circ_0071271 & $8.50 \pm 1.39$ & $8.43 \pm 1.32$ & 0.56 & 0.577 \\
\hline
\end{tabular}
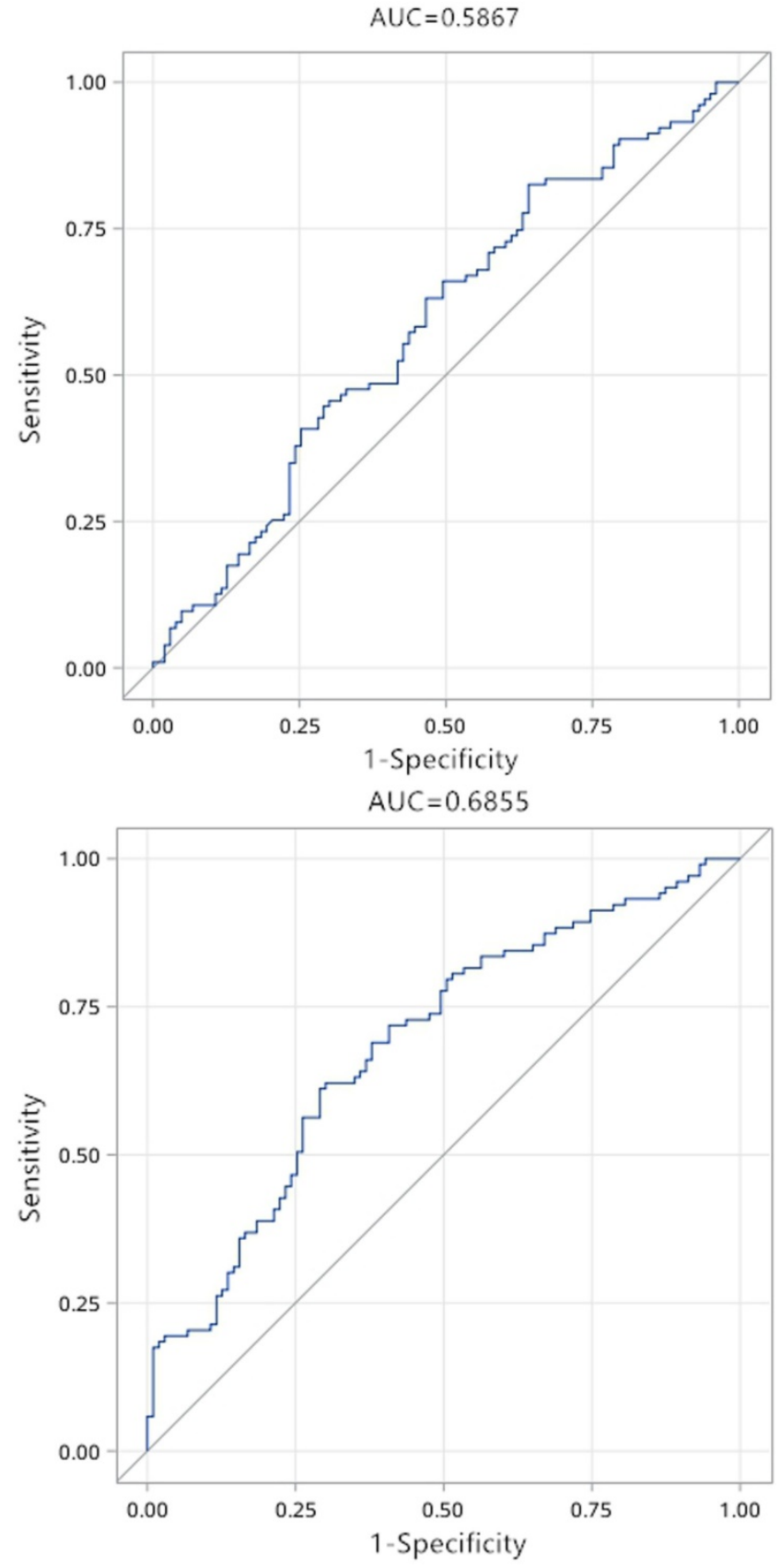

Figure 4. ROC curve of hsa_circ_0071106 in diabetes. Note: The picture on the top is the ROC curve before adjustment, and the picture on the bottom is the ROC curve after adjusting the influencing factors such as occupation, blood lipid (AUC $<0.5$ has no diagnostic value, $A \cup C>0.5$ is diagnostic value).

\section{The diagnostic value of differentially expressed circRNA in diabetes}

The results of Q-PCR verification showed that only the expression difference of hsa_circ_0071106 in diabetes among the selected candidate circRNAs had statistical significance. To determine the diagnostic values of hsa_circ_0071106 for type 2 diabetes, ROC curve analysis was performed (Figure 4). Using the grouping of diabetes as the state variable and the relative expression level of hsa_circ_0071106 as the 
test variable to draw the ROC curve. The results in Table 5 show that the sensitivity was $82.5 \%$, the specificity was $35.9 \%$ and AUC was 0.587 , suggesting that hsa_circ_0071106 have diagnostic value in the diagnosis of diabetes. According to the maximum Youden index, the best cutoff point for exploring has circ_0071106 was 5.914. After adjusting occupation and blood lipid influencing factors, the AUC increased to 0.690 , the sensitivity was $69.0 \%$, and the specificity was $62.1 \%$, and its diagnostic value was improved compared with that before adjusting the risk factors. According to the largest Youden index, the best cut-off point of hsa_circ_0071106 was 6.243. From the results, it could be seen that occupational, blood lipid and other factors should be adjusted for the differentially expressed circRNA, and a higher diagnosis accuracy and reliability could be obtained.

Table 5. Diagnostic value of differentially expressed circRNA in diabetes

\begin{tabular}{llllll}
\hline circRNA & AUC & sensitivity & specificity & Cut off & Youden \\
\hline hsa_circ_0071106 (After) & 0.587 & 0.825 & 0.359 & 5.914 & 0.184 \\
hsa_circ_0071106 (Later) & 0.690 & 0.621 & 0.699 & 6.243 & 0.320 \\
\hline
\end{tabular}

\section{Multivariate logistic regression analysis of hsa_circ_0071106 and diabetes}

The factors that were meaningful in the analysis of the single-factor logistic regression model were incorporated into the multi-factor logistic regression model. After correction for occupation and blood lipids; table 6 showed the results of the multi-factor Logistic regression model, which indicated that occupation, hyperlipidemia and hsa_circ_0071106 had a stronger correlation with diabetes $(P<0.05)$. The high expression level hsa_circ_0071106 was a risk factor and the high expression level of hsa_circ_0071106 was 2.819 (95\% CI: 1.415 5.616) times higher than the low expression level of hsa_circ_0071106.

\section{Predict miRNA by circRNA}

Import the raw data of hsa_circ_0071106 into miRNA target gene prediction software such as TargetScan and miRanda, predict five miRNAs that may be combined with hsa_circ_0071106. The results showed that the targets of miRNA bound to has circ_0071106 were hsa-miR-6830-3p, hsa-miR-4743-3p, hsa-miR-2682-3p, hsa-miR-1206 and hsa-miR-29a-5p.

\section{hsa_circ_0071106 function prediction analysis}

In order to predict the function of hsa_circ 0071106, we conducted a GO analysis. In the display of the results in Figure 5: the MF process showed that hsa_circ_0071106 was mainly enriched in the active elements of "protein binding", the CC process was mainly enriched in cells such as "nucleus and cytoplasm", and the BP process is mainly enriched in the process of "transcription, DNA template".

\section{hsa_circ_0071106 path prediction analysis}

In KEGG pathway analysis, the target genes of hsa_circ_0071106 were enriched with $P<0.05$. The results showed that the differentially expressed hsa_circ_0071106 was related to "MAPK signaling pathway" (Table 7).

\section{Discussion}

This study revealed the expression levels of circRNAs in the peripheral blood of T2DM patients was significantly different from that of healthy subjects. A total of 798 circRNAs were detected, and the circRNA expression profile of diabetic blood samples was preliminarily constructed. According to the differential expression analysis, it was found that the expression levels of 86 circRNAs in diabetic group and healthy group were statistically significant. According to volcano and scatter plots, 70 circRNAs were up-regulated in diabetes, and 16 circRNAs were down-regulated. In these 86 circRNA differential expression profiles, we found that hsa_circ_0071106 had a statistically significant difference. To remove other influencing factors, the ROC curve showed that the AUC of hsa_circ_0071106 was 0.690. With a sensitivity of $62.1 \%$ and a specificity of $69.9 \%$, showed that hsa_circ_0071106 could be considered as a biomarker for the diagnosis of T2DM.This result was consistent with Liu's.

Table 6. Multivariate logistic regression of hsa_circ_0071 106 and diabetes

\begin{tabular}{|c|c|c|c|c|c|c|}
\hline Variable & $\beta$ & $\mathrm{S}$ & $z$ & $P$ & OR $(95 \% \mathrm{CI})$ & After OR $(95 \% \mathrm{CI})$ \\
\hline \multicolumn{7}{|c|}{ Occupation } \\
\hline No & 1.000 & - & - & - & - & - \\
\hline Yes & -1.030 & 0.307 & 11.268 & $<0.001$ & $0.350(0.190 \sim 0.643)$ & $0.357(0.196 \sim 0.651)$ \\
\hline \multicolumn{7}{|c|}{ Hyperlipidemia } \\
\hline No & 1.000 & - & - & - & - & - \\
\hline Yes & 0.948 & 0.396 & 5.741 & 0.017 & $2.800(1.360 \sim 5.764)$ & $2.581(1.188 \sim 5.604)$ \\
\hline \multicolumn{7}{|c|}{ hsa_circ_0071106 } \\
\hline Low & 1.000 & - & - & - & - & - \\
\hline High & 1.036 & 0.352 & 8.687 & 0.003 & 2.478 (1.306 4.701) & $2.819(1.415 \sim 5.616)$ \\
\hline
\end{tabular}


Table 7. KEGG pathway enrichment analysis of hsa_circ_ 0071106

\begin{tabular}{lll}
\hline KEGG signaling pathway & Number of genes $(\%)$ & $P$ \\
\hline MAPK signaling pathway & $12(2.4)$ & 0.039 \\
Melanoma & $7(1.8)$ & $6.7 \times 10^{-3}$ \\
Osteoclast differentiation & $7(1.4)$ & $9.2 \times 10^{-3}$ \\
Mucin type O-Glycan biosynthesis & $4(0.8)$ & 0.037 \\
\hline
\end{tabular}

However, what is the specific mechanism of action of hsa_circ_0071106 in T2DM? Through bioinformatics analysis technology, we use Targetscan, miRanda and other databases to predict five miRNA targets: hsa-miR-6830-3p, hsa-miR-47433p, hsa-miR-2682-3p, hsa-miR-1206 and hsa-miR-29a$5 \mathrm{p}$, which were combined with the hsa_circ_0071106. Then the predicted target genes were analyzed by GO and KEGG pathway through DAVID online software, and the biological processes and possible signal pathways of hsa_circ_0071106 were preliminarily analyzed, which provided effective information for further research of hsa_circ_0071106.

Among the five miRNA targets predicted by hsa_circ_0071106, hsa-miR-29a-5p (miR-29a) has been verified by many cell models and animal experiments

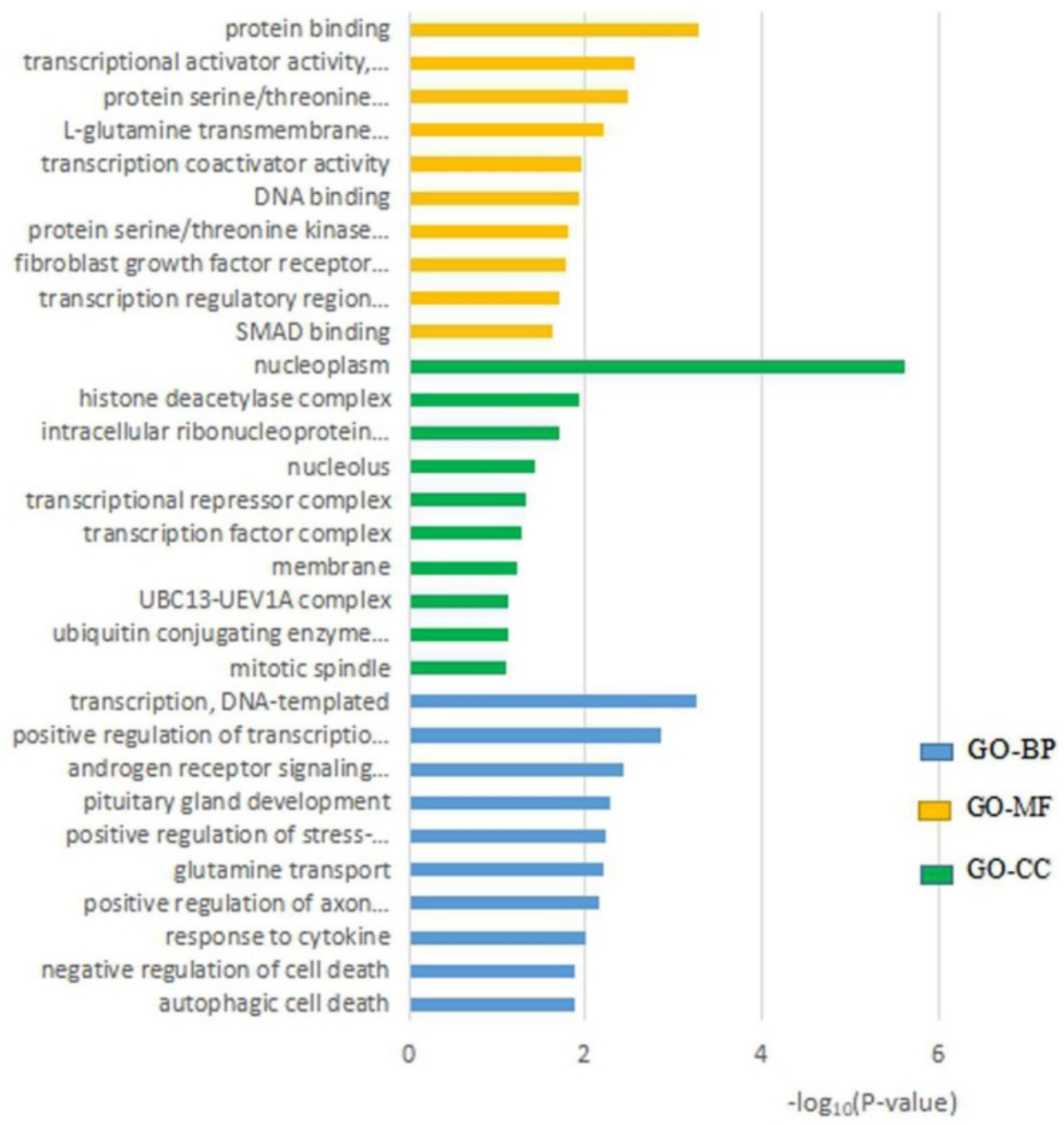

Figure 5. The top 10 significant $\mathrm{GO}$ entries of hsa_circ_0071106. related to diabetes and complications. Wang Lei [21] studied that up-regulation of miR-29a in rat liver tissue caused down-regulation of $\mathrm{FOXO3}$, which led to insulin resistance. Yang [22] revealed that miR-29a regulates the body's insulin secretion and glucose metabolism. MiR29a inhibits the expression of insulin receptor base 1 (IRS-1) during translation by acting on the 3'untranslated region (3'-UTR) of IRS-1. A large amount of reduced IRS-1 will damage the insulin signal transduction and glucose absorption of muscle cells. In addition, studies have confirmed that the decreased expression level of IRS-1 is involved in the pathogenesis of T2DM [23-25]. Aibin He [26] found that the presence of miRNA-29a/miRNA $-29 b$ in adipocytes would impede the absorption of glucose. When miRNA-29 is at a high level, it will inhibit the activation of Akt and cause insulin resistance. At this time, the internal environment is in High sugar levels, and the high sugar internal environment will promote the expression of miRNA-29a/miRNA-29b, making the condition of diabetes worse. Therefore, studies have shown that hsa-miR-29a-5p (miR-29a) may affect the pathogenesis of diabetes through the way of causing insulin resistance. The enrichment results of GO analysis showed that hsa_circ_0071106 may mainly exist in the nucleoplasm, bind to a certain protein, or participate in the process of transcribed genes. The KEGG pathway results show that hsa_circ_0071106 with differential expression is related to the MAPK signaling pathway, which is an important pathway in the cell signal transduction system [27] and one of the pathways that affect insulin signaling [28]. The MAPK signaling pathway includes four extracellular signal pathways including extracellular signal-regulated kinase (ERK), p38 mitogen-activated protein kinase (p38MAPK), jnk amino terminal kinase (JNK), and ERK5/BMK1 [27]. ERK, p38MAPK, and JNK signaling pathways are involved in regulating insulin transduction. The ERK signaling pathway is involved in the regulation ofIRS-1. Liu [29] and others have studied that insulin sensitivity is affected by the activity of ERK1/2MAPKs. When the activity increases, insulin sensitivity decreases. Yoo 
[30] found that the JNK signaling pathway promoted phosphorylation of the PTB segment S307 of IRS-1. The role of the p38MAPK signaling pathway in diabetes was different from that of ERK and JNK. The p38MAPK signaling pathway could increase the expression of glucose transporter 4 (GluT4) proteins, and the smooth transport of GluT4 could reduce the body's insulin resistance or T2DM. However, recent studies have also found that p38MAPK could accelerate cell proliferation and increase protein deposition under the action of high glucose and transforming factor (TGF- $\beta$ ), which could lead to complications such as diabetic nephropathy or diabetic vascular disease. This study found that the expression level of hsa_circ_0071106 was higher in T2DM group, so the overexpression of hsa_circ_0071106 may play a role in promoting the onset of diabetes. In addition, after the enrichment of the KEGG pathway, it has been shown that hsa_circ_0071106 is related to the transduction of the MAPK signaling pathway. It can be speculated that hsa_circ_0071106 may be involved in the process of JNK-mediated phosphorylation of IRS-1, or may be involved in the process of ERK regulating IRS-1; which in turn affects blood glucose metabolism balance. To sum up, the function prediction results showed that hsa_circ_0071106 was involved in biological processes such as protein binding or regulation of gene transcription, or MAPK signaling pathway that regulates insulin signal transduction, or may be involved in the pathway of hsa-miR-29a-5p regulating diabetes.

The limitations of this study were as follows: 1 . in this study, only 4 circRNAs were selected from the circRNA differential expression profiles for Q-PCR verification of the population. For other differentially expressed circRNAs, no further population verification was done. 2 . The bioinformatics analysis in this study only predicts the functional mechanism of circRNA with differential expression, and has not been verified by cell and animal experiments. 3. This study was a single-center study. The living environment of the research subjects was the same. Whether the circRNA gene expression profile was applicable to other populations was unclear. Therefore, further research could be conducted by collecting people from different regions for verification.

\section{Acknowledgements}

\section{Financial support}

We greatly appreciate financial support from the following organizations:
- The People's Republic of China, Guangdong Natural Science Foundation (2018A030313435, 2018A0303130249);

- Guangdong Basic and Applied Basic Research Foundation (No. 2020A1515010073);

- Guangdong provincial science and technology research project of traditional Chinese medicine (20181060);

- The Open Project of Metabolic Disease in Guangdong Province of Combine Traditional Chinese and Western Medicine Research Center (201701).

\section{Competing Interests}

The authors have declared that no competing interest exists.

\section{References}

1. Leehey DJ, Zhang JH, Emanuele NV, et al. BP and Renal Outcomes in Diabetic Kidney Disease: The Veterans Affairs Nephropathy in Diabetes Trial. Clinical Journal of the American Society of Nephrology Cjasn. 2015; 10(12): 2159-2169.

2. Shanghai Diabetes Research Collaboration Group. Survey Report on Diabetes among 100,000 Population in Shanghai Area. Journal of Shanghai First Medical College. 1980; 02: 137-138.

3. Bao Xuguang, Wang Zhongwei, He Yan et al. Study on the imbalance pattern of intestinal flora in T2DM and diabetic nephropathy. Chinese Journal of Laboratory Medicine. 2019, 42(6): 469-478.

4. Chen $\mathrm{X}$, Huang Jin. Current status and influencing factors of diabetes cognition in inpatients with diabetes. Modern Medicine and Health, 2016, (14): 2146-2148

5. Su Wei, Wang Li, Shang Youquan, et al. Observation of abnormal glucose metabolism in fasting plasma glucose, oral glucose tolerance test and glycated hemoglobin screening. Chinese Journal of Diabetes. 2013, 21(2): 154-156.

6. Physician WGC. Diagnosing diabetes mellitus in clinical practice: is fasting plasma glucose a good initial test? (p89-93). Practical Diabetes International. 2010, 18(3): 89-93.

7. Tabák AG, Herder C, Rathmann W, et al. Prediabetes: A High-Risk State for Diabetes Development. Lancet, 2012, 379(9833): 2279-2290.

8. Xiong W, Luo Y, Liu H, et al. The peripheral blood circularRNA profile in patients with ST-segment elevation myocardial infarction. Journal of Clinical Cardiology, 2018.34(4):343-347.

9. Zhang I, Xu Y, Xu S, et al. Plasma Circular RNAs, Hsa circRNA 025016, Predict Postoperative Atrial Fibrillation After Isolated Off-Pump Coronary Artery Bypass Grafting. Journal of the American Heart Association Cardiovascular Cerebrovascular Disease.2018,7(2): e006642.

10. Lin SP, Ye S, Long Y, et al. Circular RNA expression alterations are involved in OGD/R-induced neuron injury. Biochemical Biophysical Research Communications, 2016, 471(1):52-56.

11. Lukiw WJ. Circular RNA (circRNA) in Alzheimer's disease (AD). Frontiers in Genetics, 2013, 4(4): 307-315.

12. Li $\mathrm{P}$, Chen $\mathrm{S}$, Chen $\mathrm{H}$, et al. Using circular RNA as a novel type of biomarker in the screening of gastric cancer. Clinica Chimica Acta, 2015, 444: 132-136.

13. Siegel RL, Miller KD, Jemal A. Cancer statistics, 2017. CA:A Cancer Journal for Clinicians,2017,67(3):177-193

14. Jae Hoon B, Qing $Z$, Feng L, et al. The landscape of microRNA, Piwi-interacting RNA, and circular RNA in human saliva. Clinical Chemistry, 2015, 61(1): 221-230

15. Memczak S, Jens M, Elefsinioti A, et al. Circular RNAs are a large class of animal RNAs with regulatory potency. Nature,2013,495(7441): 333-338.

16. Jeck WR, Sorrentino JA, Wang $K$, et al. Circular RNAs are abundant, conserved, and associated with ALU repeats. RNA,2013,19(2): 141-157.

17. Suzuki H, Tsukahara T. A view of pre-mRNA splicing from RNase R resistant RNAs. International Journal of Molecular Sciences, 2014, 15(6): 9331-9342.

18. Chen LL. The biogenesis and emerging roles of circular RNAs[J]. Nat Rev Mol Cell Biol, 2016, 17(4): 205-211.

19. Abouhaidar MG, Venkataraman S, Golshani A, et al. Novel coding, translation, and gene expression of a replicating covalently closed circular RNA of 220 nt. Proceedings of the National Academy of Sciences of the United States of America,2014,111(40): 14542-14547.

20. Chinese Medical Association Diabetes Branch. Guidelines for the prevention and treatment of T2DM in China (2017 edition). Chinese Journal of Practical Internal Medicine, 2018, 38(04): 34-86 
21. Wang Lei, Qin Jiugang, Wang Chuan. Effect of Ophiopogon japonicus extract on the expression of miRNA-29a and FOXO3 in liver tissue of insulin resistant rats. Chinese Journal of Experimental Pharmacology, 2018, 24(1): 132-137.

22. Yang W-M, Jeong H-J, Park S-Y, et al. Induction of miR-29a by saturated fatty acids impairs insulin signaling and glucose uptake through translational repression of IRS-1 in myocytes. FEBS letters, 2014, 588(13): 2170-2176.

23. Szendroedi I, Phielix E, Roden M. The role of mitochondria in insulin resistance and T2DM mellitus. Nature Reviews Endocrinology, 2012, 8(2): 92-98

24. Carvalho E, Jansson P-A, Nagaev I, et al. Insulin resistance with low cellular IRS-1 expression is also associated with low GLUT4 expression and impaired insulin-stimulated glucose transport. The FASEB journal, 2001, 15(6): 1101-1103.

25. Rondinone CM, Wang L-M, Lonnroth $\mathrm{P}$, et al. Insulin receptor substrate (IRS) 1 is reduced and IRS- 2 is the main docking protein for phosphatidylinositol 3-kinase in adipocytes from subjects with non-insulin-dependent diabetes mellitus. Proceedings of the National Academy of Sciences, 1997, 94(8): 4171-417

26. He A, Zhu L, Gupta N, et al. Overexpression of micro ribonucleic acid 29, highly up-regulated in diabetic rats, leads to insulin resistance in 3T3-L1 adipocytes. Molecular endocrinology, 2007, 21(11): 2785-2794.

27. Gong Xiaowei, Jiang Yong. Research progress of mitogen-activated protein kinase-activated protein kinase (MK). Advances in Biochemistry and Biophysics, 2007, 34(7): 695-701.

28. Yang Xi, Liu Yujie, Xing Hanying, et al. Regulation of mitogen-activated protein kinase signaling pathway on the expression of peroxisome proliferator-activated receptor $\gamma$-coactivator in muscle cells cultured with palmitic acid . China Diabetes Journal, 2017, (11): 62-66

29. Liu Z, Patil IY, Jiang T, et al. High-fat diet induces hepatic insulin resistance and impairment of synaptic plasticity. J PloS one, 2015, 10(5): e128274.

30. Yoo JY, Kim TH, Kong S, et al. Role of Mig-6 in hepatic glucose metabolism[J]. Journal of Diabetes, 2016, 8(1): 86-97. 